\title{
Crescimento em Goniopsis cruentata (Latreille) (Crustacea, Brachyura, Grapsidae) em laboratório
}

\author{
Zilanda de Souza Silva ${ }^{1}$ \\ Lídia Miyako Yoshii Oshiro ${ }^{1}$
}

\begin{abstract}
Growth in Goniopsis cruentata (Latreille) (Crustacea, Brachyura, Grapsidae) in laboratory. The growth of the crab Goniospis cruentata (Latreille, 1803), was studied in the laboratory, through of the molt increment and intermolt period. A total of 86 crabs ( 48 males and 38 females) were collected in the Itacuruçá's Mangrove in Mangaratiba's county, Rio de Janeiro. The crabs were maintened in the laboratory in four 10001 tanks, with continuous water circulation during fifteen months. They were fed each two days with pieces of fish and fruits. The crabs varied in size from 19.3 to $56.0 \mathrm{~mm}$ in males and 23.3 to $48.8 \mathrm{~mm}$ in females. The males presented until four molts during the experiment and the females until six molts. The molts occurred during all the months. The mean percentage of molt increment was 6.1 $\pm 2.7 \%$, for both sexes and it decreased with the animal growth. The relation between the molt increment and the carapace width can be expressed by the equation $\mathrm{IM}=-$ $0.32 \mathrm{LC}+18.26(\mathrm{r}=-0.82)$. This relation didn't show any significative difference between the sexes. The mean intermolt period was of $87.6 \pm 37.3$ days in male crabs and $97.3 \pm 27.6$ days in females. The growth was contiguous, through successive molts, although the increment was reduced with animal size. The intermolt period was proportional to the animal size increase, however males with autotomy of limbs had the intermolt period reduced.

KEY WORDS. Brachyura, Goniopsis cruentata, growth, molt increment, intermolt period, laboratory
\end{abstract}

Os crustáceos apresentam um padrão de crescimento diferente dos demais animais, por apresentarem vários estágios de desenvolvimento da fase larval até a fase adulta e o crescimento através de mudas sucessivas. A muda em crustáceos pode ser descrita como o processo de eliminação da carapaça velha ou exoesqueleto, com secreção de um novo, fazendo parte do mecanismo de crescimento (LOBÃO et al. 1996). Durante a pré-muda um novo tegumento é formado, na muda o tegumento velho é liberado, o crustáceo aumenta em tamanho pela absorção de água e na pós-muda o novo tegumento endurece pelo processo de calcificação (HARTNOLL 1988). A rigidez do tegumento facilita a mensuração permitindo então o estudo do crescimento dos crustáceos a cada muda. A porcentagem de aumento no tamanho do animal entre a pré-muda e a pós-muda é o incremento de muda e o tempo gasto entre duas mudas sucessivas é o período de intermuda. Esses dois parâmetros determinam a taxa de crescimento nos crustáceos (HARTNOLL 1988).

Estudos sobre o incremento de muda em braquiúros foram realizados por HARTNOLL $(1982,1988)$ e, especificamente nos grapsídeos, destacam-se os traba-

1) Estação de Biologia Marinha, Universidade Federal Rural do Rio de Janeiro. Rua Sereder, 23860-000 Itacuruçá, Mangaratiba, Rio de Janeiro, Brasil. E-mail: oshiro@ufrrj.br 
lhos realizados em Pachygrapsus crassipes Randall, 1839 (OLMSTED \& BAUMBERGER 1923; HIATT 1948); Hemigrapsus oregonensis (Dana, 1851) e Hemigrapsus nudus (Dana, 1851) (OLMSTED \& BAUMBERGER 1923); Aratus pisonii (H. Milne Edwards, 1837) (WARNER 1967); e Sesarma cinereum (Bosc, 1817) e Sesarma reticulatum (Say, 1802) (SEIPLE \& SALMON 1987).

Quanto ao período de intermuda foram realizados estudos nas lagostas Panulirus argus (Latreille, 1804) (TrAVIS 1954); Nephrops norvegicus Linnaeus, 1758 (SARDÀ 1991); e Homarus vulgaris Milne-Edwards, 1896 (HEWETT 1974); no camarão Macrobrachium borelli (Nobili, 1896) (SETZ \& BUCKUP 1977); e nos grapsídeos Pachygrapsus crassipes (HIATT 1948) e Aratus pisonii (WARNER 1967).

Goniopsis cruentata (Latreille, 1803) é um caranguejo semiterrestre da família Grapsidae, conhecido popularmente como aratu ou mulata. Essa espécie se distribui no Atlântico Ocidental, nas Bermudas, Flórida, Golfo do México, Antilhas, Guianas, Brasil (Fernando de Noronha e do Pará a Santa Catarina) e no Atlântico Oriental do Senegal até Angola (Melo 1996). É uma espécie de importância econômica na Região Nordeste do Brasil, e nos manguezais da Baía de Sepetiba sendo muito abundante (OSHIRO et al. 1998).

Trabalhos realizados com essa espécie, ainda são escassos, destacando-se: WARNER (1970) que estudou o comportamento durante encontros intraespecíficos; YOUNG (1973), ZANDERS (1978), ZANDERS \& MARTELO (1986, 1987) e BURGGREN et al. (1993) que realizaram trabalhos sobre a fisiologia. No Brasil, esta espécie foi estudada por COBO \& FRANSOZO (1998) sobre o crescimento relativo e COBO \& FRANSOZO (1999) sobre os aspectos reprodutivos.

Portanto, este trabalho teve como objetivo contribuir para o conhecimento desta espécie, estudando o crescimento de $G$. cruentata em laboratório, através do incremento de muda e período de intermuda.

\section{MATERIAL E MÉTODOS}

Um total de 86 indivíduos de Goniopsis cruentata foi coletado no Manguezal de Itacuruçá, Mangaratiba, Rio de Janeiro, Brasil durante duas coletas, novembro/98 (grupo I-26 machos e 24 fêmeas) e fevereiro/99 (grupo II-22 machos e 14 fêmeas). A captura foi manual com auxílio de um puçá.

Os caranguejos foram transportados para o Laboratório da Estação de Biologia Marinha da Universidade Federal Rural do Rio de Janeiro(EBM/UFRRJ), onde foram mantidos durante quinze meses. No laboratório, a largura da carapaça (LC) de cada animal foi mensurada com paquímetro de precisão $(0,1 \mathrm{~mm})$. Posteriormente, os animais foram marcados individualmente, com ferro aquecido e colocados em quatro tanques de $1000 \mathrm{l}$. Os tanques tinham a parte central mais elevada, permitindo que esta permanecesse seca, e as bordas com fluxo contínuo de água do mar, com salinidade média de $34 \%$, variando de 30 a $37 \%$. A temperatura e o fotoperíodo utilizados foram os do ambiente.

Os animais foram alimentados a cada dois dias, com pedaços de peixes e frutas.

Os tanques foram observados diariamente em relação a ocorrência de autotomia e regeneração dos apêndices perdidos, e os indivíduos que sofreram muda, foram devidamente mensurados e anotados a largura da carapaça antes e após a muda. 
O incremento de muda foi expresso pela porcentagem do aumento da largura da carapaça após a muda [(LC pós-muda - LC pré-muda)/LC pré-muda] x 100 . O período de intermuda foi determinado pelo tempo gasto em dias, entre duas mudas consecutivas.

O percentual de sobrevivência total dos animais ao final do experimento foi de 6,7\% (Machos: Grupo I - 3,4\%; Grupo II - 13\%; Fêmeas: Grupo I-4,5\%; Grupo II $-6,7 \%)$.

As relações entre o incremento de muda e o tamanho do animal e o período de intermuda e o tamanho do animal foram comparadas em animais que realizaram o processo de autotomia e regeneração em ambos os sexos, através do Teste "t" de Student, a nível de 5\% de significância.

\section{RESULTADOS E DISCUSSÃO}

O tamanho dos animais variou de 19,3 a 56,0 $\mathrm{mm}$ de largura da carapaça, apresentando o tamanho médio de $35,64 \pm 7,84 \mathrm{~mm}$. O tamanho médio de fêmeas e machos, apresentou diferença significativa (teste $\mathrm{t}, \mathrm{p}<0,05$ ) em ambos os grupos (Tab. I). Os animais do Grupo I foram maiores que do grupo II (Fig. 1).

Tabela I. Goniopsis cruentata. Dados morfométricos, incremento de muda, período de intermuda e autotomia dos animais em cativeiro. (LC) Largura da carapaça.

\begin{tabular}{|c|c|c|}
\hline Parâmetros & Machos & Fêmeas \\
\hline Número de individuos Grupo I & 26 & 24 \\
\hline Número de indivíduos Grupo ॥ & 22 & 14 \\
\hline Variação de LC (mm) - Grupo I & 32,0 a 56,0 & 27,0 a 48,8 \\
\hline Variação de LC (mm) - Grupo II & 19,3 a 43,6 & 23,3 a 44,2 \\
\hline Variação de LC (mm) - Total & 19,3 a 56,0 & 23,3 a 48,8 \\
\hline Tamanho médio (mm) - Grupo I & $41,3 \pm 6,8$ & $35,4 \pm \quad 4.9$ \\
\hline Tamanho médio (mm) - Grupo ॥ & $30,6 \pm 7,7$ & $33,4 \pm$ \\
\hline Tamanho médio $(\mathrm{mm})$ - Total & $38,0 \pm 7,2$ & $36,8 \pm 6,0$ \\
\hline Número máximo de mudas & 4,0 & 6,0 \\
\hline Média de mudas & $2,3 \pm 0,8$ & $2,6 \pm 1,5$ \\
\hline Variação do incremento de muda (\%) & 1,9 a 12,5 & 2,1 a 11,5 \\
\hline Média do incremento de muda $(\%)$ & $6,0 \pm 2,9$ & $6,0 \pm 2,6$ \\
\hline Variação do periodo de intermuda (dias) & 48 a 208 & 37 a 133 \\
\hline Média do período de intermuda (dias) & $87,6 \pm 37,2$ & $93,3 \pm 27,6$ \\
\hline Individuos que realizaram autotomia (\%) & 66,7 & 46,6 \\
\hline Variação do periodo de intermuda (dias) em animais sem autotomia & 48 a 208 & 37 a 127 \\
\hline Variação do periodo de intermuda (dias) em animais com autotomia & 49 a 133 & 42 a 133 \\
\hline Média do período de intermuda (dias) em animais sem autotomia & $108,1 \pm 51,8$ & $93,7 \pm 28,4$ \\
\hline Média do periodo de intermuda (dias) em animais sem autotomia & $77,3 \pm 23,3$ & $99,3 \pm 27,2$ \\
\hline
\end{tabular}

Do total de caranguejos, 40 indivíduos ( 20 machos e 20 fêmeas) realizaram pelo menos uma muda, obtendo-se um total de 102 mudas, que ocorreram durante todo o período de estudo (Figs 2 e 3 ). Os machos realizaram até quatro mudas, com uma média de 2,5 $\pm 0,8$ mudas durante o período do experimento. A média nas fêmeas foi 2,6 $\pm 1,5$ mudas, mas houve fêmeas realizando até seis mudas. O número médio de mudas realizado em machos e fêmeas não diferiu significativamente (teste $\mathrm{t}, \mathrm{p}>0,05)$. 

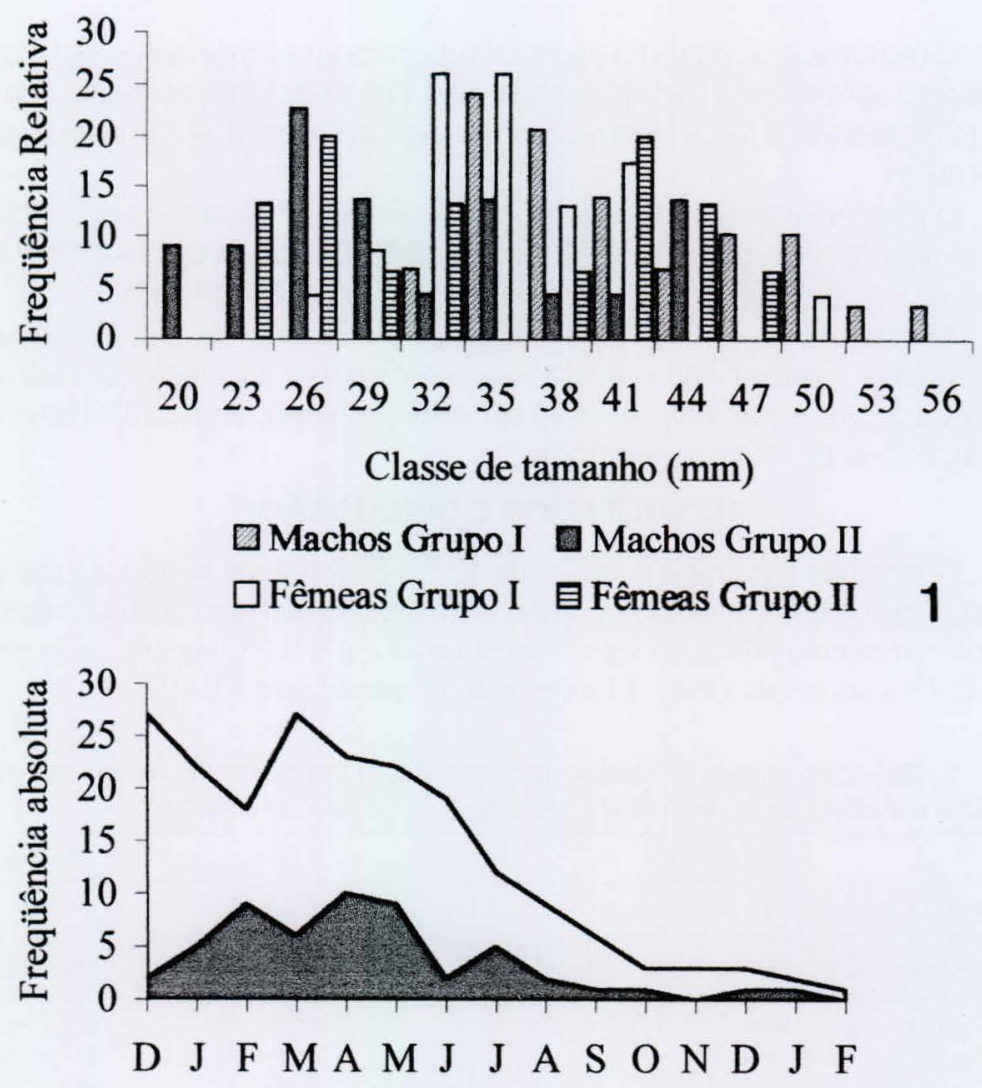

Meses

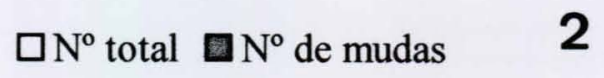

Figs 1-2. Goniopsis cruentata. (1) Distribuição da freqüência relativa dos animais em cativeiro em relação a classe de tamanho (Grupo I novembro/1998, Grupo II fev/99); (2) distribuição da freqüência absoluta mensal de machos e o número de mudas realizadas em cativeiro no periodo de novembro/1998 a fevereiro/2000.

A atividade de muda ocorreu durante todos os meses, porém os meses que apresentaram maior ocorrência de muda em ambos os sexos foram os de fevereiro a maio (Figs 2 e 3 ).

A ocorrência de mudas sucessivas nos indivíduos mantidos em laboratório foi possibilitada pelo fluxo contínuo de água nos tanques. Segundo BLISS (1982), os crustáceos decápodos marinho ou dulcícola absorvem muita água através da parede do intestino durante a muda, pois esses animais usam a água para aumentar seu novo exoesqueleto, alargando-o. 

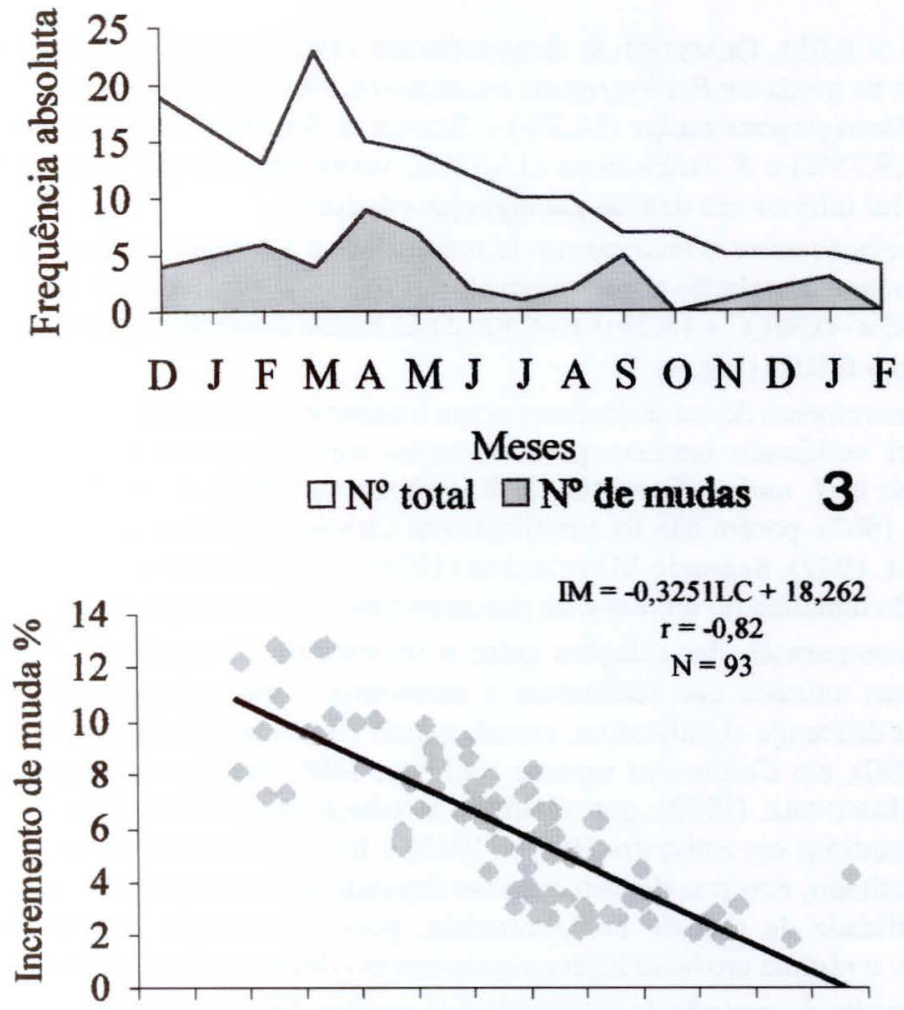

\section{7 \\ Largura da carapaça na pré-muda (mm) \\ Machos e fêmeas}

Figs 3-4. Goniopsis cruentata. (3) Distribuição da freqüência absoluta mensal de fêmeas e o número de mudas realizadas em cativeiro no período de novembro/1998 a fevereiro/2000; (4) relação entre o incremento de muda (IM) e a largura da carapaça (LC) nos animais (machos e fêmeas) em cativeiro no período de novembro/1998 a fevereiro/2000.

Alguns animais durante o experimento, tiveram seus apêndices perdidos, isto é, realizaram a autotomia. Essa autotomia em cativeiro, pode ter ocorrido por doença, defesa, comportamento reprodutivo, stress do animal ou durante o processo de muda.

A porcentagem de indivíduos que realizaram autotomia foi maior nos machos 66,7\%, enquanto nas fêmeas foi de 46,6\%. SPIVAK \& POlitis (1989) verificaram em Cyrtograpsus angulatus Dana, 1851 uma incidência maior de autotomia em fêmeas adultas do que em machos, porém o trabalho foi realizado com os animais no ambiente natural.

A porcentagem média do incremento de muda em $G$. cruentata, foi $6,1 \pm$ $2,7 \%$ (Tab. I), não havendo diferença significativa desse incremento entre os sexos 
(teste $\mathrm{t}, \mathrm{p}>0,05)$. OlmSTED \& BAuMBerger (1923) verificaram a média do incremento de muda em Pachygrapsus crassipes (8,5\%), Hemigrapsus oregonensis (12,2\%), Hemigrapsus nudus (14,2\%) e SEIPLE \& SALMON (1987) em Sesarma cinereum $(9,75 \%)$ e $S$. reticulatum $(11,67 \%)$, verificando-se que a média em $G$. cruentata foi inferior aos demais caranguejos citados.

A relação entre o incremento de muda (IM) e a largura da carapaça (LC), apresentou uma correlação negativa em ambos os sexos, podendo ser descrita pela equação $\mathrm{IM}=-0,32 \mathrm{LC}+18,26(\mathrm{r}=-0,82)$ e não houve diferença significativa entre os sexos ( $p>0,001)$ (Fig. 4).

O incremento de muda decresceu com o aumento de tamanho do animal (Fig. 4), que foi verificado também para as outras espécies como $P$. crassipes, $H$. oregonensis e H. nudus (OLMSTED \& BAUMBERGER 1923), e em Aratus pisonii (WARNER 1967), porém não foi verificado em Carcinus maenas (Linnaeus, 1758) (HARTNOLl 1982). Segundo MAUCHLINE (1976), o incremento de muda depende da idade, do tamanho do animal e do processo natural de variação na população.

A comparação das relações entre o incremento de muda e a largura da carapaça em animais que realizaram a autotomia e em animais intactos, não apresentou diferença significativa, corroborando com o resultado encontrado por SMITH (1990), em Callinectes sapidus Rathbun, 1896. Porém, este resultado discorda de HARTNOLL (1982), que verificou a redução do incremento em algumas espécies mantidas em cativeiro. HIATT (1948) e KURATA (1962) verificaram esse mesmo resultado, mas ressaltaram que isso depende das condições de manutenção e adaptabilidade da espécie no laboratório, pois mesmo que o ambiente seja controlado, o regime artificial limita o crescimento desses animais confinados.

A média do período de intermuda nos machos foi de $87,6 \pm 37,3$ dias e nas fêmeas de 97,3 $\pm 27,6$ (Tab. I), apresentando diferença significativa (teste t, p < $0,05)$ entre os sexos. WARNER (1967), verificou um período médio de intermuda de 53 dias em fêmeas maduras de Aratus pisonii e HIATT (1948) verificou um período entre 20 a 45 dias em Pachygrapsus crassipes.

A média do período de intermuda nos machos com autotomia foi menor do que nos machos sem autotomia, apresentando uma diferença significativa (teste t, $p<0,05$ ), mas as fêmeas não apresentaram diferença significativa (teste t, $p>0,05$ ) entre essas médias (Tab. I).

A relação entre o período de intermuda (PI) e o tamanho do animal (LC) apresentou uma correlação positiva para as fêmeas e uma fraca correlação positiva para os machos (Fig. 5). Não houve uma diferença significativa entre os sexos ( $p$ > $0,001)$.

A relação entre o período de intermuda e o tamanho do animal em machos sem autotomia apresentou uma correlação positiva e nos machos com autotomia a correlação foi fraca. Comparando-se as duas relações, verifica-se que houve uma diferença significativa ( $p<0,001$ ) (Fig. 6), demostrando que a autotomia reduziu o período de intermuda nos machos.

As relações entre o período de intermuda e o tamanho do animal em fêmeas sem autotomia e com autotomia, apresentaram uma correlação positiva. A compa- 

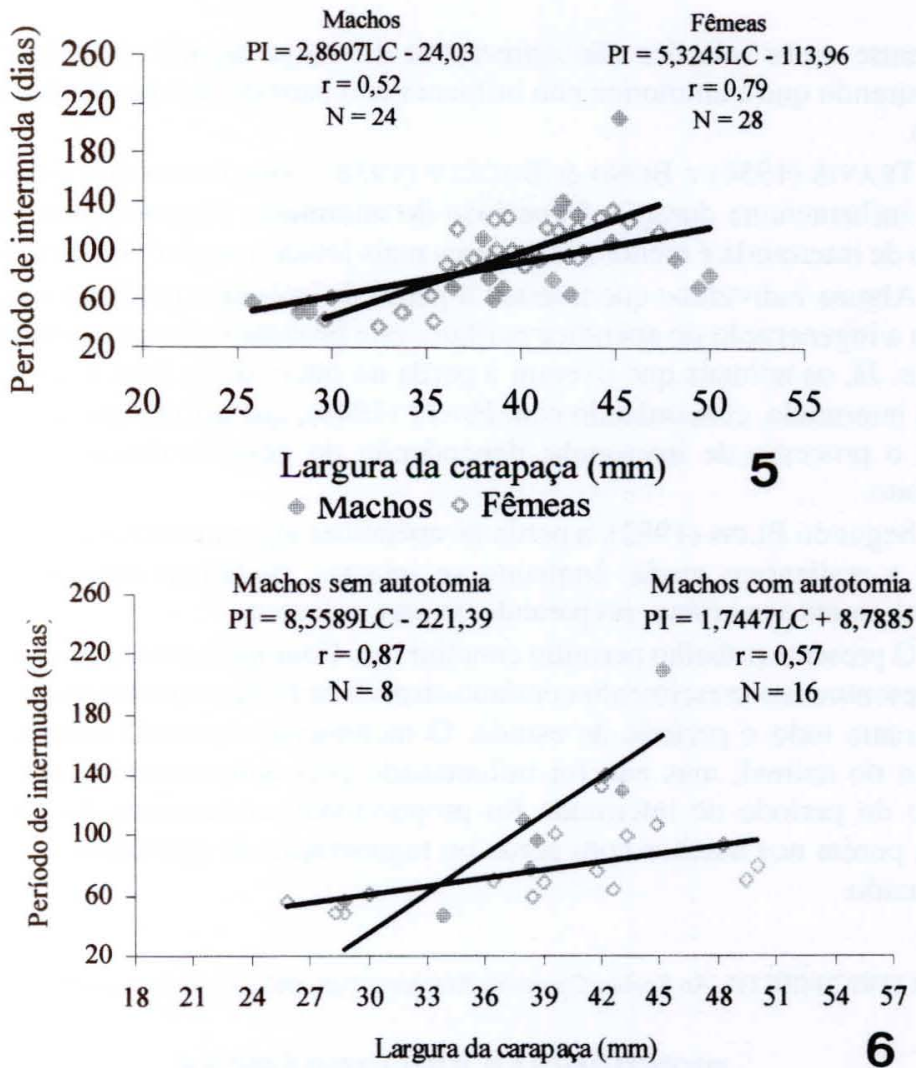

- Machos sem autotomia

Machos com autotomia

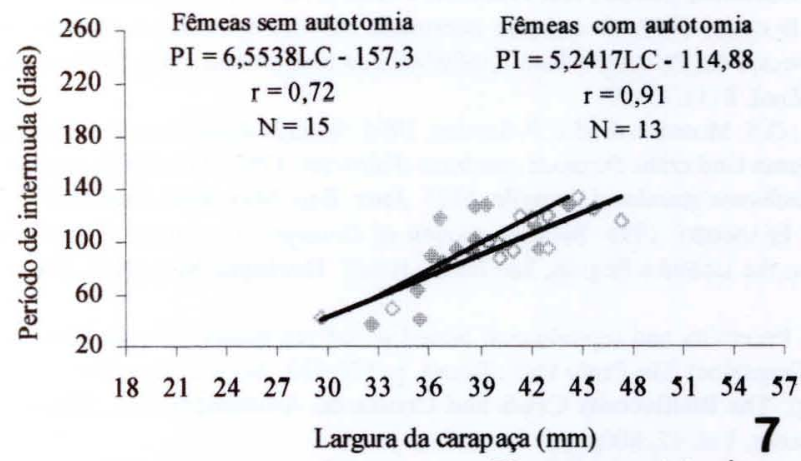

- Fêmeas sem autotomia

- Fêmeas com autotomia

Figs 5-7. Relação entre o período de intermuda (PI) e a largura da carapaça (LC) em Goniopsis cruentata em cativeiro no período de novembro/1998 a fevereiro/2000: (5) machos e fêmeas; (6) machos com apêndices intactos e machos que realizaram o processo de autotomia; (7) fêmeas com apêndices intactos e fêmeas com processo de autotomia. 
ração entre essas relações não apresentou diferença significativa ( $p>0,001$ ), demonstrando que a autotomia não influenciou o período de intermuda em fêmeas (Fig. 7).

TRAVIS (1954) e BOND \& BUCKUP (1988), observaram que o tamanho do animal influencia na duração do período de intermuda. Segundo estes autores, o período de intermuda é menor nos animais mais jovens e maior nos de maior porte.

Alguns indivíduos que tiveram a perda do apêndice próximo à muda, não fizeram a regeneração do apêndice perdido, este processo só ocorreu na intermuda seguinte. Já, os animais que tiveram a perda no início desta fase, regeneraram na própria intermuda, concordando com HiATT (1948), que afirma que a regeneração acelera o processo de intermuda, dependendo do grau de desenvolvimento do tegumento.

Segundo BLISS (1982), a perda de apêndices algumas vezes pode induzir as fêmeas a realizarem muda, enquanto se intactas, poderiam estar realizando o desenvolvimento ovariano, preparando-se para a desova.

O presente trabalho permitiu concluir que Goniopsis cruentata em laboratório, apresentou um crescimento contínuo através de mudas sucessivas, que ocorreram durante todo o período de estudo. O incremento de muda diminuiu com o tamanho do animal, mas não foi influenciado pela autotomia dos apêndices. A duração do período de intermuda foi proporcional ao aumento do tamanho do animal, porém nos machos com perda ou regeneração de apêndices, esse período foi reduzido.

AGRADECIMENTO. Ao Senhor Casemiro Antonio Alves, pelo auxílio nas coletas.

\section{REFERÊNCIAS BIBLIOGRÁFICAS}

BuIss, D.E. 1982. Shrimps, lobsters and crabs. New Jersey, New Centry Publishers, 241p.

Bond, G., \& L. BuCKUP. 1988. O ciclo da intermuda em Macrobrachium borellii (Nobili, 1896) (Crustacea, Decapoda, Palaemonidae): a influência da temperatura e do comprimento do animal Revta bras. Zool. 5 (1): 45-59.

BURGgren, W.W.; G.S. Moreira \& M.C.F. SANTOS. 1993. Specific dynamic action and the metabolism of the brachyuran land crabs Ocypode quadrata (Fabricius, 1787), Goniopsis cruentata (Latreille, 1803) and Cardisoma guanhumi Latreille, 1825. Jour. Exp. Mar. Biol. Ecol. 169: 117-130.

Сово, V.J. \& A. Fransozo. 1998. Relative growth of Goniopsis cruentata (Crustacea, Brachyura, Grapsidae), on the Ubatuba Region, São Paulo, Brazil. Iheringia, Sér. Zool., Porto Alegre (84): 21-28.

1999. Fecundity and reproduction period of the red mangrove crab Goniopsis cruentata (Brachyura, Grapsidae) São Paulo state, Brazil, p. 527-533. In.: J.C. von VAupel KLeIN \& F.R. Schram (Eds). The Biodiversity Crisis and Crustacea. Amsterdam, A.A. Balkema Publishers, Crustacean Issues, Vol. 12, 800p.

Hartnoll, R.G. 1982. Growth, p. 111-196. In: L.G. AbelE (Ed.). The Biology of Crustacea. New York, Academic Press, Vol. 2, 443p.

- 1988. Growth and molting, p.186-210. In: W.W. Burggren \& B.R. MCMAhon (Ed.).

Biology of the land crabs. New York, Cambridge Univ. Press, 446p.

HewETT, C.J. 1974. Growth and mouting in the common lobster (Homarus vulgaris Milne-Eduards).

Jour. mar. Biol. Ass. U.K. 54: 379-391.

Revta bras. Zool. 19 (3): 915 - 923, 2002 
HiATT, R.W. 1948. The biology of the lined shore crab, Pachygrapsus crassipes Randall. Pacif. Sci. 2: 135-213.

Kurata, H. 1962. Studies on the age and growth of Crustacea. Bull. Hokkaido reg. Fish. Res. Lab. 24: $1-115$.

Lobão, V.L.; E.A. Roverso; M. Lace \& E. Hortencio. 1996. Ciclo de muda e crescimento em Macrobrachium amazonicum Heller, 1862 e Macrobrachium rosenbergii De Man (Decapoda, Palaemonidae). Bol. Inst. Pesca, São Paulo, 23: 35-45.

Mauchline, J. 1976. The Hiatt growth diagram for Crustacea. Mar. Biol., Heidelberg, 35: 79-84.

Melo, G.A.S. 1996. Manual de identificação dos Brachyura (caranguejos e siris) do litoral brasileiro. São Paulo, Editora Plêiade, 603p.

OLMSTED, J.M.D. \& J.P. BAUMBERGER. 1923. Form and growth of grapsoid crabs. A comparison of the form of three species of grapsoid crabs and their growth at molting. Jour. Morph., Hoboken, 38: 279-294.

OshiRo, L.M.O.; R. Silva \& Z.S. Silva. 1998. Composição da fauna de braquiúros (Crustacea, Decapoda) dos Manguezais da Baía de Sepetiba - RJ. Nauplius, Rio Grande, 6: 31-40.

SARDÀ, F. 1991. Reproduction and moult synchronism in Nephrops norvegicus (L.) (Decapoda, Nephropidae) in the Western Mediterranean: is spawning annual or biennial? Crustaceana, Netherlands, 60 (2): 186-199.

SEIPLE, W. \& M. SALMON. 1987. Reproductive, growth and life-history contrasts between two species of grapsid crabs, Sesarma cinereum and S. reticulatum. Mar. Biol., Heidelberg, 94: 1-6.

SETZ, E.Z.F. \& L. BuCKuP. 1977. A duração da intermuda e o comportamento reprodutivo de Macrobrachium borellii (Nobili, 1896) e Palaemonetes (Palaemonetes) argentinus Nobili, 1901 no cultivo em laboratório (Crustacea, Decapoda, Palaemonidae). Rev. Brasil. Biol., Rio de Janeiro, 37 (4): 899-906.

SмiтH, L.D. 1990. Patterns of limbs loss in the blue crab, Callinectes sapidus Rathbun, and the effects of autotomy on growth. Bull. Mar. Sci., Miame, 46: 23-36.

SPIVAK, E.D. \& M.A. PoLITIS. 1989. High incidence of limb autotomy in a crab population from a coastal lagoon in the province of Buenos Aires, Argentina. Can. Jour. Zool. 67: 1976-1985.

Travis, D.F. 1954. The molting cycle of the spiny lobster, Panulirus argus Latreille. I. Molting and growth in laboratory-maintained individuals. Biol. Bull., Masschusetts, 107 (3): 433-450.

WARNER, G.F. 1967. The life history of the mangrove tree crab. Aratus pisonii. Jour. Zool. London 153: $321-325$.

-1970. Behavior of two species of grapsid crab during intraspecific encounters. Behavior, Leinden, 1: 9-19.

YounG, R.E. 1973. Responses to respiratory stress in relation to blood pigment affinity in Goniopsis cruentata (Latreille) and (to a lesser extent) in Cardisoma guanhumi Latreille. Jour. Exp. Mar. Biol. Ecol., Amsterdam, 11: 91-102.

ZANDERS, I.P. 1978. Ionic regulation in the mangrove crab Goniopsis cruentata. Comp. Biochem. Physiol., New York, 60: 293-302.

ZANDERS, I.P. \& M.J. MARTELO. 1986. Influence of temperature on ionic regulation in the mangrove crab Goniopsis cruentata. Comp. Biochem. Physiol., New York, 78 (2): 249-254.

Zanders, I.P. \& M.J. Martelo. 1987. Contribution of $\mathrm{Na} / \mathrm{Na}$ and $\mathrm{Cl} / \mathrm{Cl}$ exchanges to sodium and chloride fluxes in the crab Goniopsis cruentata. Jour. Comp. Physiol. B. Biocheml Syst. Environ Physiol., New York, 157 (5): 599-606.

Recebido em 10.XII.2001; aceito em 04.IX.2002. 